\title{
10th AIAA/ISSMO Multidisciplinary Analysis and Optimization Conference, Albany, NY, 30 August - 1 September 2004
}

\author{
A. Messac
}

Key words Multidisciplinary Design Optimization (MDO), Multidisciplinary Analysis and Optimization (MA\&O)

The Multidisciplinary Analysis and Optimization (MA\&O) Conference is jointly sponsored by the American Institute of Aeronautics and Astronautics (AIAA) and the International Society of Structural and Multidisciplinary Optimization (ISSMO). It will be held in the scenic Saratoga Springs and Lake George area of New York State, USA.

We are approaching the twenty-year anniversary of the biennial MA\&O international conference, which brings together industry, government, and academia to present and discuss the latest in MA\&O technology. Critical recent developments have placed MA\&O in a central role in multidisciplinary design: (i) the ground-breaking advances in non-deterministic approaches, (ii) the dramatically reduced cost and increased speed of computing, and (iii) the vibrant continued development of MA\&O technology. These developments have also resulted in a broadening of the application of MA\&O - to areas well beyond traditional structural aerospace systems.

This international conference will take a retrospective and prospective look at MA\&O, and allow for a solid assessment of the state of the art. Contributions are invited covering a broad set of applications, from traditional aerospace and automotive to consumer products design. Methodological approaches of interest range from multidisciplinary and multiobjective to multiscale methods. Applications and MA\&O approaches that address micro, nano, and biotechnology are also of particular interest. Topics of interest also include non-deterministic approaches, human-machine interaction, computational infrastructure, and supporting software and hardware.

Proposals for panels and special sessions are also welcome. Those addressing new methodologies as well as new classes of MA\&O applications are particularly sought.

Further information regarding the conference can be obtained at the following web address:

http://www.rpi.edu/ messac/mao-2004

Published online: 16 October 2003

(C) Springer-Verlag 2003

\section{A. Messac}

Multidisciplinary Design and Optimization Laboratory, Dept. of Mechanical, Aerospace, and Nuclear Engineering, Rensselaer Polytechnic Institute, Troy, New York, 12180, USA e-mail: messac@rpi.edu 